\title{
Backside Lift-Out Specimen Preparation: Reversing the Analysis Direction in Atom Probe Tomography
}

\author{
T.J. Prosa, D. Lawrence, D. Olson, D.J. Larson, and E.A. Marquis* \\ Imago Scientific Instruments, Madison, WI 53711 \\ *Department of Materials, University of Oxford, Oxford OX1 3PH, United Kingdom
}

The field of atom probe tomography (APT) has seen a rapid expansion in the types of materials and device structures that can be analyzed via advances in pulsed-laser atom probe technology [1] and adoption of focused ion beam (FIB) and lift-out specimen preparation techniques originally developed for transmission electron microscopy (TEM). Standard APT lift-out preparation [2] first requires removal of a wedge of material containing the region-of-interest (ROI), which often includes a near-surface region of the wedge. Subsequent annular FIB milling [3] creates the sharp tip required for APT. Analysis proceeds from the vacuum interface down into the bulk of the wedge with the original surface perpendicular to the tip axis. By using an axial rotation manipulator [4], specimens may be positioned and shaped to allow arbitrary orientations of the ROI.

It is well known that the three-dimensional reconstruction in APT can be affected and distorted by changes in the tip surface shapes that deviate from that of a single curvature surface [5]. Since an arbitrary ROI usually is composed of regions with differing evaporation fields, the tip shape almost always deviates from that of a single curvature surface. In order to better understand potential structural/composition artifacts resulting from these shape distortions, a method has been developed to prepare samples in a complementary "bottom-up" or backside orientation.

Backside lift-out specimen preparation is the process of taking a surface or near-surface ROI and placing it upside down on a wedge of user-constructed material for subsequent preparation into an APT tip. The overall approach is shown schematically in Fig. 1. First, the surface ROI, is buried below $\sim 2$ microns of sputtered metal that serves as the bulk of the wedge once it is rotated 180 degrees (50 nm nickel, 2 microns silver, $50 \mathrm{~nm}$ nickel). Second, a pyramid of FIB-deposited platinum is used to increase the amount of material between the ROI and the eventual mount to a carrier structure (in this case a tungsten needle mounted in a $3 \mathrm{~mm}$ grid geometry compatible with TEM and atom probe, Figs. 2 and 3). The topology of the platinum deposit mimics the angled shape of the bottom of a top-down wedge [2], aiding in the attachment of the wedge to the carrier structure. Third, the wedge is trimmed so that the top surface is flat and appropriately separated from the ROI. Fourth, the wedge is rotated 180 degrees and properly mounted to a carrier structure. Finally, annular milling is performed to create the final tip shape for APT analysis. This procedure has been successfully performed on a structure composed of metal multilayers on silicon [6,7], with the resulting analysis direction (arrowed in Fig. 5) being parallel (as opposed to anti-parallel) to the film growth direction. Fig. 5 illustrates these results in a composition profile ranging from silicon substrate (with thin oxide), through a Ta seed layer and into an IrMn-based antiferromagnetic layer.

[1] J. H. Bunton et al., Micro. Microanal. 13 (2007) 418.

[2] K. Thompson et al., Ultramicroscopy 107(2-3) (2006) 131.

[3] D. J. Larson et al., Ultramicroscopy 79 (1999) 287.

[4] D. Lawrence et al., Micro. Microanal. 14(Suppl 2) (2008) 1004.

[5] F. Vurpillot et al., Micro. Microanal. 10(3) (2004) 384.

[6] S. S. P. Parkin et al., Nature Materials 3 (2004) 862.

[7] Dr. P. M. Rice, IBM Almaden, is gratefully acknowledged for providing of the materials. 

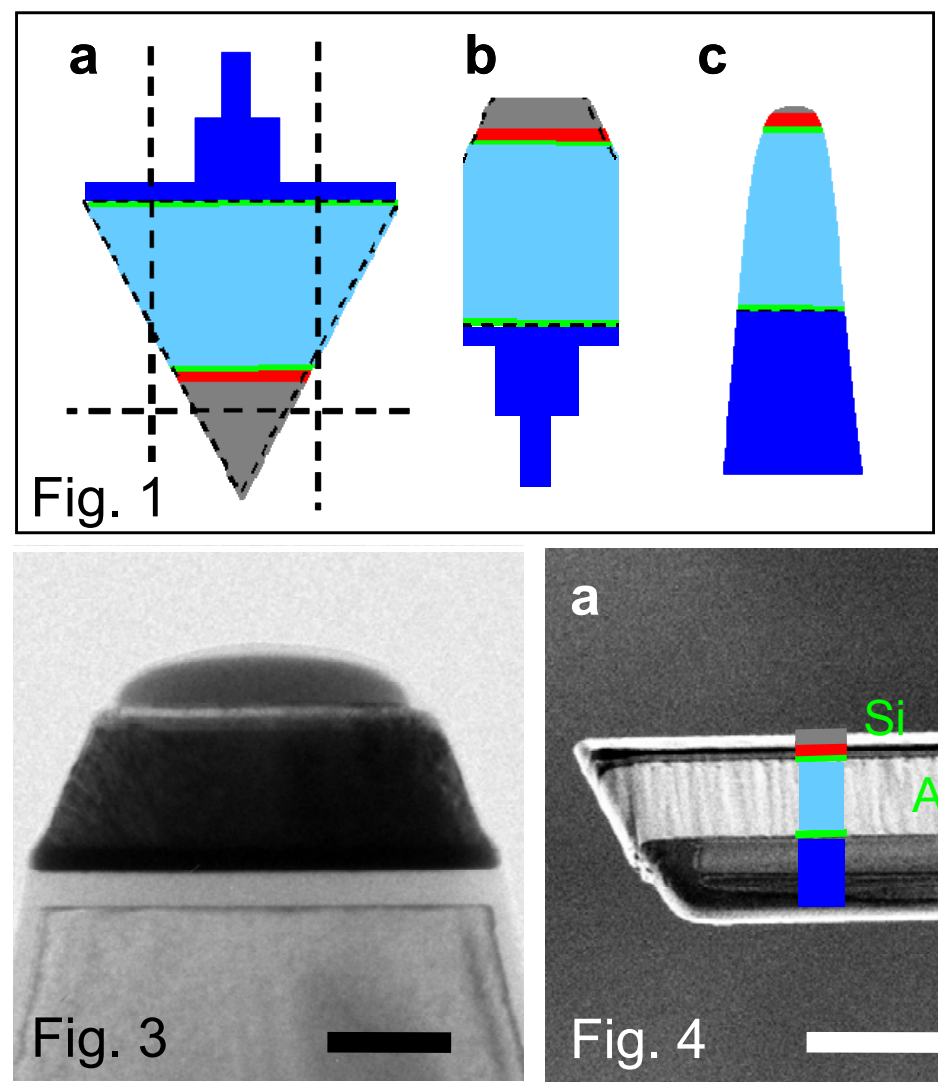
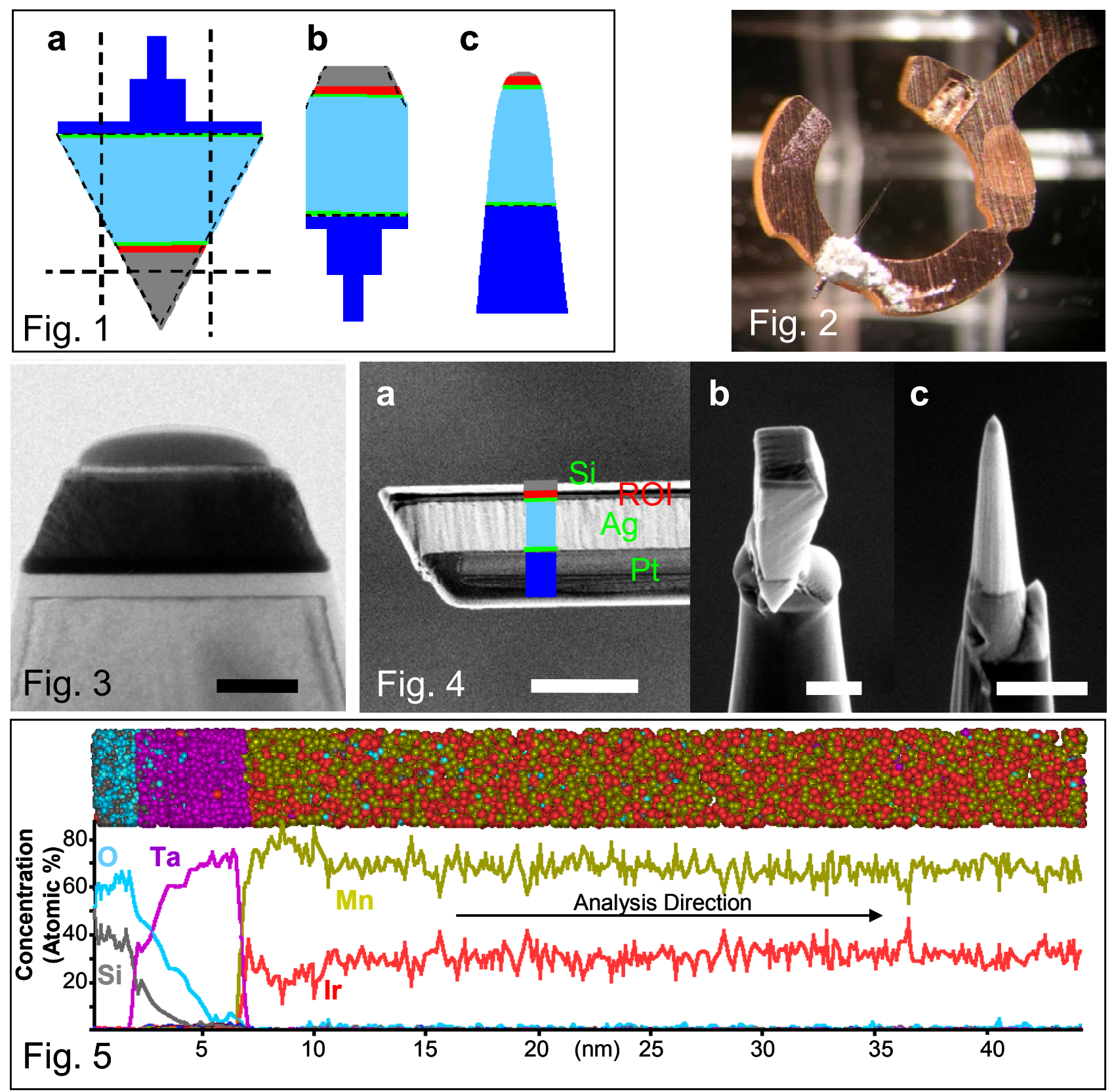

FIG. 1 Preparing a bulk material for backside analysis: (a) Composition of the wedge-above the ROI (red) is $50 \mathrm{~nm}$ nickel (green) followed by 2 microns silver (light blue), $50 \mathrm{~nm}$ nickel (green), and 1.5 microns of FIB platinum shaped in a pyramid (blue)-dashed lined indicate material to be trimmed, (b) wedge after trimming and 180 degree rotation, and (c) final tip shape after annular mill.

FIG. 2. Tungsten needle carrier structure pressed into a copper grid via the Omniprobe Short-Cut ${ }^{\mathrm{TM}}$. FIG. 3. TEM image of atom probe tip pressed into same copper grid. Note: Scale bar $40 \mathrm{~nm}$.

FIG. 4. Scanning electron microscopy images of the wedge after trimming of the sides so that the layered structure of the wedge is clearly visible (a), (b) wedge after it has been rotated, tacked to a carrier structure with FIB platinum, and the remaining wedge removed, and (c) final tip shape after annular milling. Note: Scale bars left to right 5 microns, 1 micron, and 1 micron.

FIG. 5. Atom map of the tantalum and iridium-manganese layers deposited on a silicon substrate (top), and analyzed composition as a function of depth from the backside (bottom). 